Original Research Paper

\title{
Conceptual Design, Analysis and Construction of a Fixed-Wing Unmanned Arial Vehicle for Oil and Gas Pipeline Surveillance
}

\author{
Aliyu Bhar Kisabo, Charles Attah Osheku and Sholiyi Olusegun Samuel \\ Centre for Space Transport and Propulsion (CSTP) Epe, Lagos, Nigeria
}

\author{
Article history \\ Received: 15-05-2017 \\ Revised: 25-05-2017 \\ Accepted: 01-06-2017 \\ Corresponding Author: \\ Aliyu Bhar Kisabo \\ Centre for Space Transport and \\ Propulsion (CSTP) Epe, Lagos, \\ Nigeria \\ Email: aliyukisabo@yahoo.com
}

\begin{abstract}
Oil and Gas Pipelines consists of pipes, compressors and pumps. These are frequently located in environments that are difficult to monitor and secure (e.g., creeks and remote areas). Attacks or damage to such installations can lead to enormous ecological impact and loss of revenue. Developing and implementing monitoring systems that can continuously assess the state and condition of oil and gas pipelines is very essential. Current solutions for monitoring such facilities are very manual and risky. Unmanned Aerial Vehicle (UAV) offers great new alternative solutions. In this study, we present a conceptual design of a UAV with a battery powered propulsion system for such application. Here, we show by example how a mission statement can be translated into a physical aircraft from first principle. Notably, five (5) novel mathematical equations were formulated to aid and optimise the design process. These novel equations basically relate the take-off mass of the aircraft with the wingspan, chord length and fuselage length. With such equations, for a designed take-off mass, there exist several variant of the aircraft concept by varying wingspan, chord length and fuselage length of the UAV. In addition, we used thrust-to-weight ratio in a novel approach to ensure that the power available at the propeller will be sufficient for the mission. For this phase of the design, the basic objective among others is to attain lift-off at a very short take off distance. The method proposed in this study proved to be very effective after several successful flight tests.
\end{abstract}

Keywords: Unmanned Aerial Vehicle, Lift-Off Mass, Chord Length, Wingspan, Fuselage Length, Oil and Gas Pipelines

\section{Introduction}

Nigeria is the eleventh largest producer of oil in the world and the largest in Africa. The petroleum sector contributes about $90 \%$ of the nation's foreign exchange earnings and $25 \%$ of the Gross Domestic Products. Large proportion of the Nation's oil is produced onshore and transported by extensive systems of pipelines across the Niger Delta region. These transmission pipelines comprise a network of hundreds of kilometres around the region. Pipeline networks are made up of legs of different lengths, up to thousands of kilometres and can have above- or below-ground configurations. The safety and security of all pipelines, regardless of their size, placement, or location, is of paramount importance to stakeholders and to the public. Proper maintenance of pipeline networks is also important for environmental protection.

Equipment failure along these pipeline networks such as breakage or leaks can occur for many reasons, including overage of structures and material failure, natural ground movement, accidental hot-tap and thirdparty interference or vandalism.

Nigeria lost over $\$ 11$ billion to crude oil theft and pipeline vandalization over a 4-year period from 2007 to 2011 (Idachaba, 2013). Strategies deployed by the government and these companies to tackle these challenges include the deployment of armed military personnel to these assets and along their pipeline Right of Way (ROW). These have led to huge increase in the operational expenses and a decline in revenue to both the companies and the government. The results however 
show that the deployment of these personnel and the attendant cost has not reduced the quantity and frequency of oil theft. This is because the deployment time in some of these locations also contributes to the delay in the response of the military personnel.

Large amounts of oil and gas is lost from any pipe failure and more importantly, hydrocarbon leaks are harmful to the environment through contamination and pollution that can greatly affect both humans and plants in a negative way.

Developing and implementing monitoring systems that can continuously assess the state and condition of oil and gas pipelines is essential. Furthermore, monitoring pipeline networks also involves acquiring knowledge of the impact pipelines have on the environment over time and how they affect vegetation and wildlife.

Pipeline surveillance refers to strategic monitoring of the activities on the pipeline's right of way and the adjoining strips of land whose rights are both shared by pipeline operators and landowners. Traditionally, monitoring pipeline networks has often been restricted to visual inspections or volume and mass balance measurements. Currently, the most widely used monitoring methods for oil and gas transmission pipelines are foot patrols along the pipeline route. The main disadvantage with this method is the potential for late detection of failures; when the output (oil or gas) has been reduced, or the environment has already been affected and damaged.

With the advent and progress of remote sensing technology together with image processing software, new opportunities have emerged for the development of monitoring systems with the possibility for high frequency data collection, that provide a comparatively inexpensive and spatially precise means to identify hydrocarbon leaks. Amongst the most promising techniques are Unmanned Aerial Vehicles (UAV).

The Unmanned Aerial Vehicle market has grown into a multi-billion-dollar industry, with the UAV market worth over $\$ 4.9$ billion in 2010-largely due to the growing application domain for UAVs (TGC, 2011). Unmanned systems are associated with a host of terms: Unmanned Aerial Systems (UAS), Drones, Remotely Piloted Aircraft (RPA), Unmanned Vehicle Systems (UVS) and Unmanned Airborne or Aerial Vehicles (UAV) reflecting the variety of system configurations and fields of application. Different sources use UAV or UAS as the preferred term. UAV is the term adopted by the UK Civil Aviation Authority (CAA), whilst others suggest that UAS is more correct.

An Unmanned Aerial Vehicle (UAV) is flown without a pilot on-board and is either remotely and fully controlled from another place (e.g., ground, another aircraft, space) or programmed and fully autonomous (Gomez and David, 2010). Thus, a UAV is an aircraft but an aircraft is not necessarily a UAV.

\section{Oil and Gas Pipeline Surveillance with UAV}

This solution has the capability of reducing crude oil theft by providing early accurate information to the company with respect to the bunkering activity along its pipeline ROW and enable timely deployment of personnel to contain the situation. When pressure drop is observed on any oil and gas pipeline, the operator sends a request for inspection to the facility covering that pipeline section. The UAV is then deployed to provide video and picture feedback of the situation along the pipeline in real time. The UAV also provides a scan of the entire network in the cell before returning to its base in the facility. Military personnel can then be deployed if vandal activity is detected.

\section{Advantages of Using UAV for Oil and Gas Pipeline Surveillance}

The UAV system so deployed for surveillance has advantages over the traditional method (foot patrol). Such advantages include improved mission safety, flight repeatability, the potential for reduction in operational costs and fewer weather-related flying limitations (e.g., a UAV can fly below the clouds) (Onwuka and Dike, 2015).

In phase I of this project, the conceptual design of the aerial vehicle was completed and a prototype was built and test-flown. The Autopilot necessary to carry out the mission autonomously and the integration of the ground station will be designed and integrated in phase II.

\section{Design of the UAV}

In this section, we present an approach that quickly takes a mission statement and translates it to an actual aircraft design from basic aircraft design principles. To do these effectively, we divided the UAV design into 6 major categories.

\section{Mission Statement}

Mission requirements may be in the form of point performance values (e.g., field length, turn rates, etc.), as a description of the mission profile(s), or as operational issues (e.g., payload, equipment to be carried, offensive threats, etc.) (Jenkinson and Marchman, 2003) for simplicity of design, we chose a conventional aircraft configuration. Looking at a mission that will last for about 20 min we opted for a battery based propulsion system for the UAV. Also, a short distance take-off aircraft is highly desirable. The mission flight profile will be that of a simple steady climb then cruise (at about an altitude of $150 \mathrm{~m}$ ) around the target area and finally steady decent to land.

For aerial surveillance, our UAV will basically carry a camera with wireless capability of transmitting live video and picture images in real time to a mobile ground station. Hence, the payload of the UAV is a wireless camera system. We now proceed to select an off-the shelf wireless camera system that can perform such task. 


\section{Estimating the Take-off Mass}

The total weight of the selected camera (with accessories) that can transmit high resolution pictures and videos from an altitude of about $150 \mathrm{~m}$ selected is $600 \mathrm{~g}$.

To have an estimate of the aircraft weight at take-off, it is useful to consider the historical values of the Payload Weight Fraction given as (Austin, 2010):

$$
\frac{W_{p l}}{W_{0}}=0.201
$$

In this case, our payload is:

$$
W_{p l}=0.6 \mathrm{~kg}
$$

It is now possible to estimate an initial value of the weight at take-off of the proposed UAV from (1), this gives:

$$
W_{0}=\frac{W_{p l}}{0.201}=\frac{0.6}{0.201}=3 \mathrm{~kg}
$$

Now that we have estimated the total take-off mass of the UAV, it is necessary to share it amongst the various components of the aircraft.

\section{Mass Distribution}

With Fig. 1 in perspective, we now must do a mass distribution for the various subsystems of the UAV (Giuseppe, 2008). This is necessary to ensure that at the end of the design we do not end up with a lift-off mass greater that was initially considered.

\section{Estimating Wing Surface Area, Wingspan, Chord} length and Fuselage length

From the study of comparative UAVs able to perform the same mission, a choice for Wing Load for our design was selected as:

$$
\frac{W}{S}=94 N / m^{2}
$$

Once the Wing Load and the Take-off Weight are selected, the Wing Surface Area is computed as:

$$
S_{w}=\frac{W_{0}}{W / S}=\frac{3 \times 9.81}{94}=0.312 \mathrm{~m}^{2}
$$

It is well documented in literature that the wing area is related to the wingspan and chord length as:

$$
S_{w}=b \cdot c_{w}
$$

For a rectangular wing (Sadraey, 2010), the following expressing holds:

$$
A R_{w}=\frac{b_{w}^{2}}{S_{w}}
$$

After selecting a value of Aspect Ratio (AR), the wing span and chord can now be computed from (6) and (7) respectively. We chose $A R_{w}$ of 5 and from (6) we get:

$b_{w} \cdot c_{w}=0.312 m^{2}$

Substituting (6) in (7) we get:

$$
A R_{w}=\frac{b_{w}^{2}}{b_{w} \cdot c_{w}}
$$

With (8) in perspective we can write:

$$
\begin{aligned}
& 5=\frac{b_{w}^{2}}{0.312} \\
& b_{w}=1.3 m
\end{aligned}
$$

From (8), we compute the chord length as:

$$
c_{w}=\frac{0.312}{1.3}=0.24 m
$$

Length of the UAV's fuselage is determined by a ratio of the wingspan (Bronz, 2014), this is given as:

$L=\eta b_{w}$

where, $\eta=0.8$ and $b_{w}=1.3 \mathrm{~m}$.

We computed our fuselage length by coming up with a coefficient from historical data of tail draggers (SIG Mfg. Co.,), as given in Table 1. Hence for our aircraft, the fuselage length is:

$$
L=0.8 \times 1.3=1 m
$$

\section{Novel Unified Take-off Mass Equations}

From experimentations during construction at, we came to a staggering realization that the take-off mass of an aircraft varies for different wingspan, chord length and fuselage length. This we termed the unified mass equations. To illustrate this mathematical, we will begin by substituting (5) in (6), this gives:

$$
\frac{W_{0}}{W / S}=b_{w} \cdot c_{w}
$$




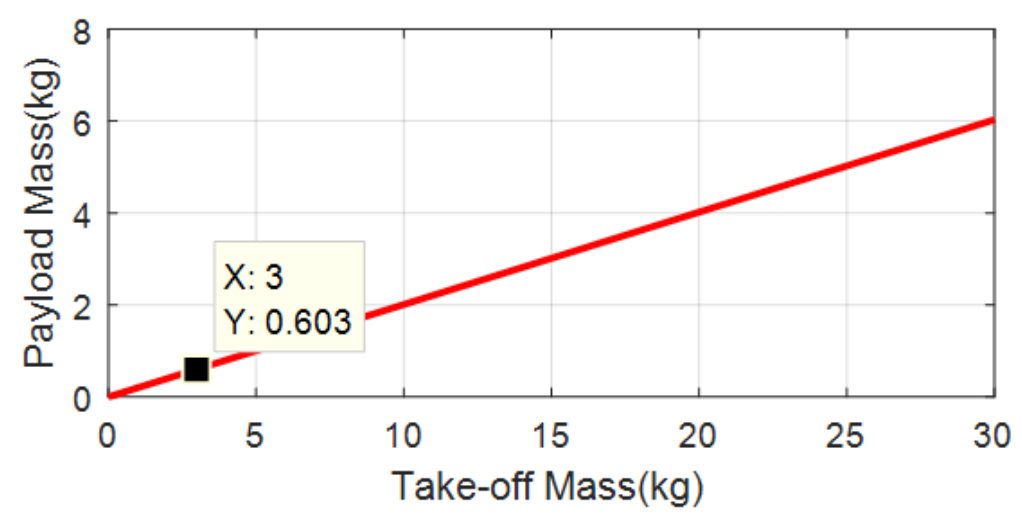

Fig. 1. Take-off mass against mass of payload

Table 1. Length and wingspan of Tail draggers

\begin{tabular}{lllll}
\hline S/N & Aircraft & $\mathrm{b}(\mathrm{m})$ & $\mathrm{L}(\mathrm{m})$ & $\mathrm{L} / \mathrm{b}(\eta)$ \\
\hline 1 & Rascal EP-49 ARF & 1.3 & 0.8 & 0.6 \\
2 & Rascal 72 EG ARF & 1.8 & 1.3 & 0.7 \\
3 & Rascal 110 ARF & 2.8 & 1.9 & 0.7 \\
4 & 4- Star 54 EG ARF & 1.3 & 1.2 & 0.9 \\
5 & 4- Star 64 EG ARF & 1.6 & 1.4 & 0.9 \\
6 & T-CLIPS EP ART & 1.5 & & 0.7 \\
& Average & & & 0.8 \\
\hline
\end{tabular}

From (14) we can express the chord length and wingspan as a function of the take-off mass as given in (15) and (16) respectively:

$c=\frac{W_{0}}{W / S} \cdot \frac{1}{b_{w}}$

$b=\frac{W_{0}}{W / S} \cdot \frac{1}{c_{w}}$

Thus, the fuselage length of the UAV as a function of the take-off mass is obtained by substituting (16) in (12):

$$
L=0.8\left\{\frac{W_{0}}{W / S} \cdot \frac{1}{c_{w}}\right\}
$$

Considering (16), since our design for this study is for $3 \mathrm{~kg}$ lift-off mass and for the selected wing loading of $94 \mathrm{~N} / \mathrm{m}^{2}$ we can express our wingspan as:

$b_{w}=\frac{0.319}{c_{w}}$

From (12) wingspan of the aircraft is expressed as:

$$
b_{w}=\frac{L}{0.81}
$$

Equating the right-hand sides of (18) and (19) we got:
$L=\frac{0.26}{c_{w}}$

The design approach for selecting aerofoil section for an aircraft is rigorous (Brusov and Petruchik, 2011), here we just go straight to mention the one we selected without given the details of the process. Our UAV will fly at subsonic speed hence, a low-speed NaturalLaminar-flow aerofoil (Ashok and Selig, 2001) section is best for our application. As such, the aerofoil section selected for the wings is the WRIGTH-6. It was patterned out with the actual design chord length size on A4 paper from the software profili (http://www.profili2.com/eng/prices.asp).

\section{Empennage Design}

The tail, like the main wing also generates lift but this lift can be neglect compared to the amount of lift produced by the main wing. The main function of the tail is to provide stability and control.

The selected aerofoil section for the empennage is NACA 0009 (Selig, 1995) also the pattern was made with the aid of profili. Empennage design (Fig. 7) begins by determining an appropriate static margin and calculating the required tail area to meet this value. Only eight variables are needed to size the horizontal and vertical tail. Table 2 outlines the empennage design variables used in this study (Struett, 2012). 
Table 2. Empennage design variables

\begin{tabular}{ll}
\hline Horizontal & Vertical \\
\hline$A R_{H}=3$ & $A R_{V}=1.3$ \\
$V_{H}=0.4$ & $V_{V}=0.04$ \\
$\lambda_{H}=1$ & $\lambda_{v}=0.6$ \\
$L_{h t}=0.6 \mathrm{~L}_{\mathrm{f}}$ & $L_{v t}=0.57 L_{f}$ \\
\hline
\end{tabular}

The tail moment arms $L_{\mathrm{ht}}$ and $L_{\mathrm{vt}}$, as depicted in Fig. 8, are the distance from the wing's 0.25 MAC to the corresponding tail's 0.25 MAC. For an aircraft which has a propeller mounted in the front the tail arm is usually set to approximately $60 \%$ of the total fuselage length.

\section{Horizontal Tail Design}

We begin here by computing the horizontal tail surface area $\left(S_{H T}\right),(21)$ gives us this required value to begin the horizontal tail design:

$S_{H_{T}}=\frac{V_{H} c_{w} S_{w}}{L_{H_{T}}}=0.049 m^{2}$

where, $S_{\mathrm{w}}=0.312 \mathrm{~m}^{2}, C_{\mathrm{W}}=0.24 \mathrm{~m}, b_{\mathrm{w}}=1.3 \mathrm{~m}$ and $L_{\mathrm{f}}$ $=1 \mathrm{~m}$.

To compute the wingspan and chord length for the horizontal tail, from (9) we can write:

$$
b_{H_{T}}^{2}=A R_{H_{T}} \cdot S_{H_{T}}
$$

From (22), $b_{\mathrm{HT}}=0.38 \mathrm{~m}$. We now compute the size of our horizontal tail chord by re-writing (6) as:

$$
c_{H_{T}}=\frac{S_{H_{T}}}{b_{H_{T}}}
$$

From (23), $C_{H T}=0.13 \mathrm{~m}$. These completes the dimensions for the horizontal tail, we now move to determine the dimensions for the vertical tail.

\section{Vertical Tail Design}

Here also, we begin by computing the vertical tail area $\left(S_{\mathrm{vT}}\right)$ as given in $(24)$. For its corresponding wingspan, we used (25) to size it:

$S_{V_{T}}=\frac{V_{V} b_{w} S_{W}}{L_{V_{T}}}=0.029 m^{2}$

$b_{V_{T}}^{2}=A R_{V_{T}} \cdot S_{V_{T}}$

From (25), $b_{\mathrm{vT}}=0.192 \mathrm{~m}$. We now obtained our vertical tail chord as:

$c_{V_{T}}=\frac{S_{V_{T}}}{b_{V_{T}}}=0.148$
Since $\lambda_{v}=0.6$, then $(26)$ is the tip chord of the vertical tail thus, the root chord for the vertical tail will be given as:

$c_{V T r}=\frac{c_{V T_{t}}}{\lambda_{v}}$

From (27), the root chord for the vertical tail was computed as $c_{V T t}=0.247 \mathrm{~m}$.

Structural details for the proposed UAV are presented in a technical drawing format as shown in Fig. 9. This is necessary to aid fabrication.

\section{Propulsion System Design}

Electric propulsion system mainly consists of four sub-elements, shown in Fig. 8; the battery, the motor controller also called as Electronic Speed Controller, (ESC), electric motor and the propeller. A gear system can also be found between the motor and the propeller but mainly it is included in the motor sub-element.

Ideally, it is required that each element in Fig. 8 be modelled and analysed before a design is made for system requirement (Gur and Rosen, 2009; Drela, 2007; Rutkay, 2014; Youngren and Chang, 2011). Since this is just the conceptual phase of the project, we will deal superficially with these elements.

Regardless of the maximum efficiency of an electric motor or a propeller, if they are not matched correctly for the given mission specifications, the resultant total efficiency will be poor (Drela, 2005). For us to quickly match propeller with motor, we used the software Moto Calc (http://www.motocalc.com/motodown.htm). The system design and performance requirements parameters in Table 3 were supplied as input to the software. Among the options for propulsion system that Moto Calc gave, the specification in Table 4 was adopted.

The maximum power the propulsion system in Table 4 will supply is 275 Watts. But, from the data sheet of (Scorpion SII-2215-1810), it suggests that for $80-100 \%$ power delivery by the propulsion system, any of the propellers in Table 5 should be used.

\section{Novel Empirical Validation of Power with Thrust- to-Weight Ratio}

For any electric driven aircraft, the propulsion system will deliver a certain amount of power. This numerical value of power is either gotten directly from datasheet of motor manufacturers, or based on test conducted in the designer's laboratory. However, there exist the need to ascertain that this power will be sufficient for the entire flight mission. As such, power output is defined as:

$\left(T-D_{a}-D_{f r}\right) V_{T O}=P_{0}$ 
Table 3. System design and performance requirements for CSTP_UAV $3.0 \mathrm{I}$

\begin{tabular}{ll}
\hline Wingspan & $1.3 \mathrm{~m}$ \\
\hline Altitude of flight & $150 \mathrm{~m}$ \\
Chord length & $0.024 \mathrm{~m}$ \\
Wing area & $0.312 \mathrm{~m}^{2}$ \\
Lift-off mass & $3.0 \mathrm{~kg}$ \\
Endurance & $30 \mathrm{~min}$ \\
Battery type & Lipo \\
Motor type & Brushless \\
\hline
\end{tabular}

Table 4. Designed propulsion system

\begin{tabular}{ll}
\hline Motor & Scorpion SII-22-810 \\
\hline Battery & $2 \times 3200$ SHD \\
Propeller & $12 \times 10$ in \\
\hline
\end{tabular}

Table 5. Datasheet propeller options for scorpion SII-22-1810

\begin{tabular}{lll}
\hline Prop. Manf & Prop size & Input voltage \\
\hline APC & $7 \times 6-$ SF & 7.4 \\
APC & $8 \times 3.8-$ SF & 7.4 \\
GEM & $8 \times 4.5-\mathrm{C}$ & 7.4 \\
GWS & $8 \times 4.3-\mathrm{SF}$ & 7.4 \\
APC & $6 \times 5.5-\mathrm{E}$ & 7.4 \\
\hline
\end{tabular}

where, $T$ is the thrust force, $D_{a}$ is the aerodynamic drag and $D_{f r}$ is the frictional drag due to the landing gears and $V_{T O}$ is the take-off velocity:

$D_{f r}=2 \mu w_{1}+\mu w_{2}$

$D_{a}=0.5 \rho V_{T O}^{2} S_{r e f} C_{D}$

Substituting (29) and (30) in (28) gives:

$\left[T-\left\{\left(0.5 \rho V_{t}^{2} S_{r e f} C_{D}\right)-\left(2 \mu w_{1}+\mu w_{2}\right)\right\}\right] V_{T O}=P_{0}$

where, $w_{1}$ and $w_{2}$ are the front back landing gears respectively and $\mu$ is the frictional coefficient of the runway, leading to:

$T=\frac{P_{0}}{V_{T O}}+\left\{\left(0.5 \rho V_{t}^{2} S_{r e f} C_{D}\right)+\left(2 \mu w_{1}+\mu w_{2}\right)\right\}$

The power system can be defined as a function of input and output power:

$$
\begin{gathered}
\eta=\frac{P_{0}}{P_{i}} \\
P_{0}=\eta P_{i}
\end{gathered}
$$

Substituting (33) in (32) we get:

$T_{i}=\frac{\eta P_{i}}{V_{T O}}+\left\{\left(0.5 \rho V_{t}^{2} S_{r e f} C_{D}\right)+\left(2 \mu w_{1}+\mu w_{2}\right)\right\}$
Each landing gear has a certain fraction of the aircraft weight acting on it through its chassis, hence we can write the following:

$$
\begin{aligned}
& w_{1}=w_{1_{t}}+w_{c h} \\
& =w_{1_{t}}+\frac{L_{1}}{L} W_{0} \\
& w_{2}=w_{2_{t}}+w_{c h} \\
& =w_{2_{t}}+\frac{L_{2}}{L} W_{0}
\end{aligned}
$$

Substituting (35) and (36) in (34) gives:

$$
\begin{aligned}
T_{i} & \left.=\frac{\eta P_{i}}{V_{T O}}+\left\{\left(0.5 \rho V_{t}^{2} S_{r e f} C_{D}\right)+\left(\begin{array}{l}
2 \mu\left(w_{1_{t}}+\frac{L_{1}}{L} W_{0}\right) \\
+\mu\left(w_{2_{t}}+\frac{L_{2}}{L} W_{0}\right)
\end{array}\right)\right)\right\} \\
& =\frac{\eta P_{i}}{V_{T O}}+\left\{\left(0.5 \rho V_{t}^{2} S_{r e f} C_{D}\right)+\left(\begin{array}{l}
2 \mu w_{1_{t}}+2 \mu \frac{L_{1}}{L} W_{0} \\
+\mu w_{2_{t}}+\mu \frac{L_{2}}{L} W_{0}
\end{array}\right)\right\}
\end{aligned}
$$

Multiplying all through with $1 / W_{0}$ and simplifying gives:

$$
\frac{T_{i}}{W_{0}}=\frac{\eta P_{i}}{W_{0} V_{T O}}+\left\{\begin{array}{l}
\frac{\left(0.5 \rho V_{T O}^{2} S_{r e f} C_{D}\right)}{W_{0}} \\
+\mu\left(2 \frac{w_{1_{t}}}{W_{0}}+\frac{w_{2_{t}}}{W_{0}}+\frac{2 L_{1}+L_{2}}{L}\right)
\end{array}\right\}
$$

For, $W_{0}=30 \mathrm{~N}, \eta=0.7, V_{T 0}=4.34 \mathrm{~ms}^{-1}, C_{D}=0.134$, $S_{\text {ref }}=0.312 \mathrm{~m}^{2}, \mu=0.02, \rho=1.225 \mathrm{kgm}^{-3}, P_{i}=220 \mathrm{~W}$ and assuming tires have masses of at most $4 \%$ of $W_{o}$, this give $0.12 \mathrm{~kg}$. The main landing gear should be between about $50-55 \%$ of the MAC computed as:

MAC Lenght $=\frac{2}{3}\left\{C_{r}+C_{t}-\frac{C_{r} C_{t}}{C_{r}+C_{t}}\right\}=0.29 m$

Hence, $55 \%$ of $M A C$ is $0.16 \mathrm{~m}$, therefore $L=L_{1}+L_{2}=$ $0.615 \mathrm{~m}, L_{1}=0.045 \mathrm{~m}, L_{2}=0.57 \mathrm{~m}$ (as presented in Fig. 11), evaluating (38) we got:

$\frac{T_{i}}{W_{0}}=1.16+0.04$

Comparing (40) with (38), the thrust to weight ratio depends on the first term in (38) -the second term is negligible. Then, we can re-write (38) as: 


$$
\frac{T_{i}}{W_{0}}=\frac{\eta_{\text {batt }} P_{i}}{W_{0} V_{T O}}
$$

where, $\eta_{\text {batt }}$ is the efficiency of the battery hence, investigate for a power input $\left(P_{i}\right)$ by substituting the value of $220 \mathrm{~W}$ as $P_{i}$ in (41) we get:

$$
\frac{T_{i}}{W_{0}}=\frac{0.7 \times 220}{30 \times 4.34}=1.07
$$

\section{Landing Gear}

The two main struts are attached to the airplane slightly ahead of the airplane's Centre of Gravity (CG).

The rudder pedals are the primary directional controls while taxing. The tail gear of the UAV attaches directly to the bottom of the rudder and extends to about $0.03 \mathrm{~m}$ below the end of the fuselage, allowing it to fit easily into the fuselage. The front landing gear is taller to ensure good clearance of propeller with ground. Each front landing gear is mounted directly onto the foam sheet to ensure that the load is transferred to a rigid part of the aircraft.

\section{Flight Test}

Aside the primary objective of acquiring technical know-how of building and flying an aircraft from scientific first principle approach indigenously, the goals for this concept design was to test several key design decisions, these are:

- To ensure that the aerofoil section selected for the wing will provide the need lift

- Taxi test to ensure adequacy of the ski to slide over of the runway (CPSU, 2013-2014)

- To ensure that the propulsion system selected will taxi the UAV sufficiently and get it to the take-off velocity

- Validate design take-off distance (less than $20 \mathrm{~m}$ )

The UAV shown in Fig. 11 was flown several times at an open field and all the concerns highlighter above was allayed.

\section{Discussion of Result}

In the next phase of this study, the payload is likely to increase in mass. This will be because of the inclusion of sensors that can detect hydrocarbon emission (because of leakage). Thus, Fig. 1 will be used to quickly determine the UAV's take-off mass. As payload mass increases, take-off mass also increase. CSTP_UAV_3.0_I was subjected to a distribution analysis as shown in Fig. 2. This mass distribution analysis was used to guide hard ware selection, especially for the propulsion system elements.

Once we have the take-off mass and mass distribution for various components in perspective we then proceeded to estimate the wing area as a function of the take-off mass of the UAV under a wind load. From Fig. 3, the wing area is expected to increase as the takeoff mass increases. This simply means that if we are considering more payloads on our UAV we must determine the exact mass increase and it must translate to more area on the wings.

The relationship between the wing area and the aspect ratio for a rectangular wing aircraft is given in (7). The UAV' chord length and wingspan were estimated as given in (10) and (11). Fuselage length of the aircraft is related to the wingspan by (12). Historical information in Table 1 of similar aircrafts, gave the relating coefficient for the determination of our fuselage length.

Standard aircraft design parameters as shown in Table 2 were used to determine the sizes of all concern variables that make up the empennage. Since it does not generate any form of lift, NACA006 was used for the aerofoil section of the horizontal and vertical tail. The aerofoil section was printed with the actual chord length from profile. This pattern was used to form ribs for both the horizontal and vertical tail.

During the initial design stages of this conceptual aircraft, we observed that a violation of the take-off mass of the UAV resulted in other anomalies in the design. This prompted the further investigation into the unique role the take-off mass plays during the design process. The results of such investigation are the novel unified take-off mass equations (15), (16) and (17). From Fig. 4, it is clearly seen that as take-off mass increases we expect that the corresponding chord length, wingspan and fuselage length should also increase. In a nutshell, an aircraft with design take-off mass of $5 \mathrm{~kg}$ will not have the same wingspan, chord length and fuselage length as an aircraft with a design take-off mass of $3 \mathrm{~kg}$. These mathematical equations simply inform us that if at the end of fabrication, we have an aircraft with mass greater than our initially designed take-off mass the aircraft design is in jeopardy and a bad one might be tempted to think of getting a more powerful propulsion system to correct this anomaly, forgetting that the load carrying capacity or lift of an aircraft is mainly a function of the wingspan and corresponding chord length. Converse is the case if the UAV's take-off mass ends up being less that the initially designed take-off mass. Hence, it is customary in aerospace industry to quest for ways of making hardware much lighter to increase overall performance of space vehicles. 


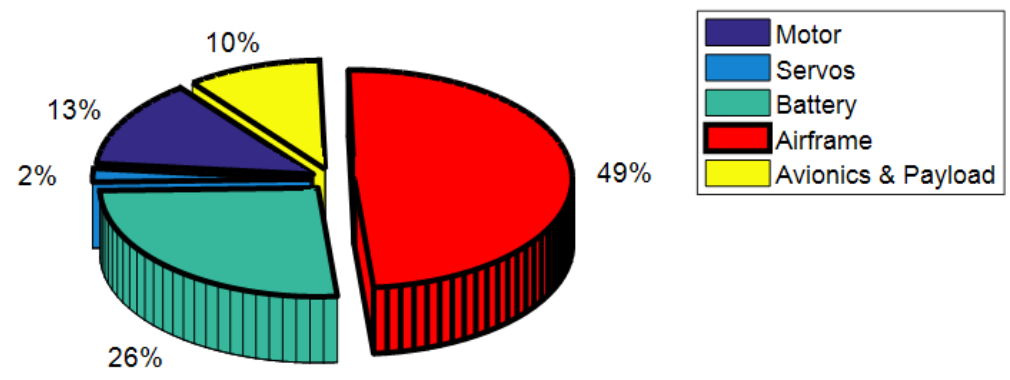

Fig. 2. CSTP UAV_3.0_I mass distribution

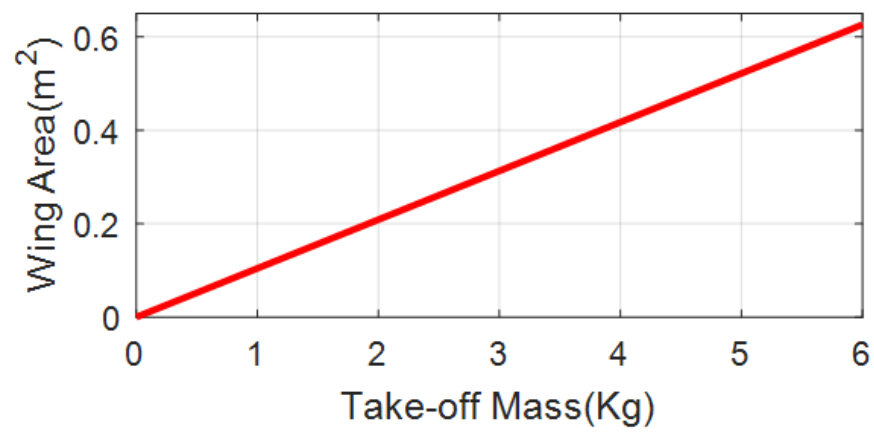

Fig. 3. Take-off mass against wing surface area

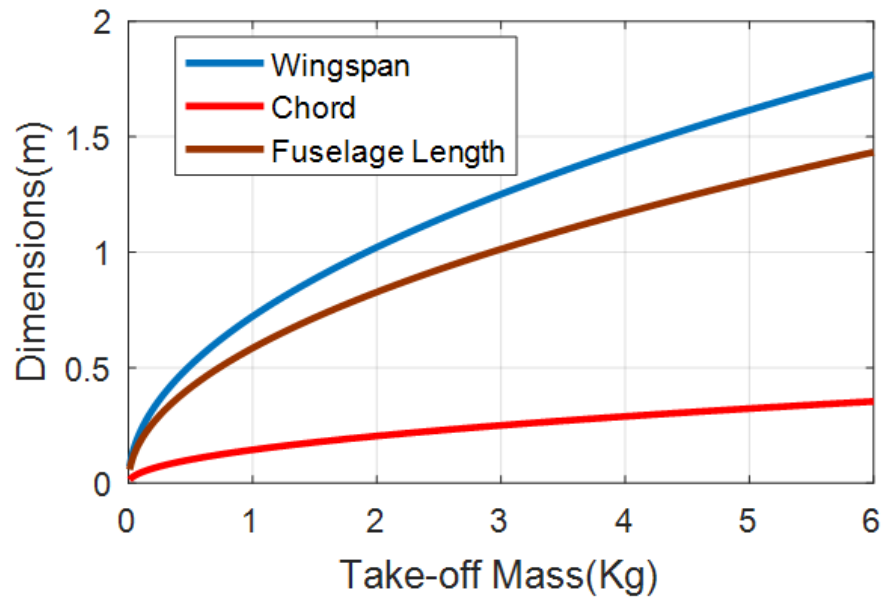

Fig. 4. Take-off mass as it affects wing span, chord length and fuselage length

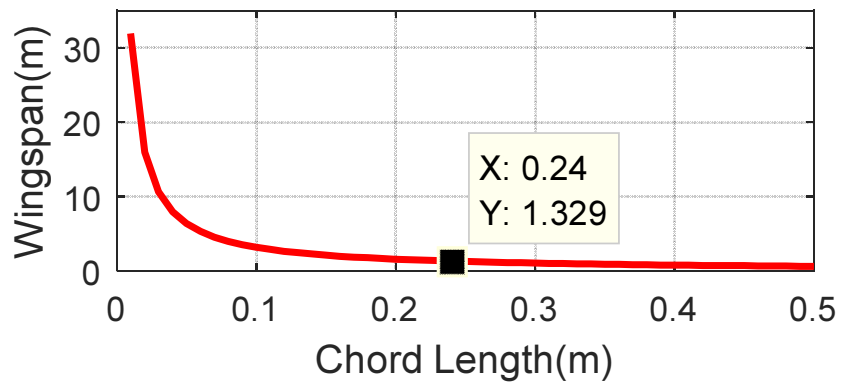

Fig. 5. Chord length against wingspan for $3.0 \mathrm{~kg}$ lift-off mass UAV 
For an electric driven aircraft, the heaviest component comes from the propulsion system (battery) Fig. 10 depicts the actual layout of the propulsion system elements in CSTP_UAV_3.0_I. To quickly design our propulsion system, information in Table 3 served as input to the software Moto Calc. Matching of all elements to form the propulsion system is invaluable, especially that of motor and propeller.

Note, from Table 4 Moto Calc recommends a $12 \times$ 10 -inch propeller to be matched with the motor Scorpion SII-22-1810. From Table 5, none of such dimension of propeller was recommended by the manufacturer of the motor. Motor used on CSTP_UAV_3.0_I wasA2212/6T with capacity $2200 \mathrm{kv}$. Manufacturers of A2212/6T recommend a $7 \times 5$ in propeller on 2 batteries of 2 cells and a $5 \times 5$ or $6 \times 4$ prop on 3 cells. Matching propeller with motor on an experimental test rig is invaluable for a UAV designer, this is necessary to validate manufacturer's claims (Brezina and Thomas, 2013). We ended up matching the motor A2212/6T with a propeller of dimension $10 \times 4$ inch. Lipo battery of capacity $4200 \mathrm{mAh}$ (3 cell) with an input voltage of 7.4 volts and a 30A Electronic Speed Controller (ESC) completed our propulsion system design. The ESC was programmed manually. This manual means of programming consists of the radio transmitter, radio receiver, the ESC and the battery connected to the motor in a loop while following series of beeping sounds to configure the system. Other options of doing this is by using Arduino microcontroller or a Programming Card.

The datasheet for A2212/6T, following manufacturer's recommendation a maximum power of $220 \mathrm{~W}$ will be delivered. Even if static and dynamic test on a test rig confirms the maximum power at the propeller, there still exists the need to ascertain that this power will be adequate for our proposed mission. To do this, we opted for an empirical method. From Fig. 12, the minimum power required which gave a thrust to weight ratio of one is 186 Watts. Thus, we need at least 186 Watts as the power output at the propeller for the entire flight mission.

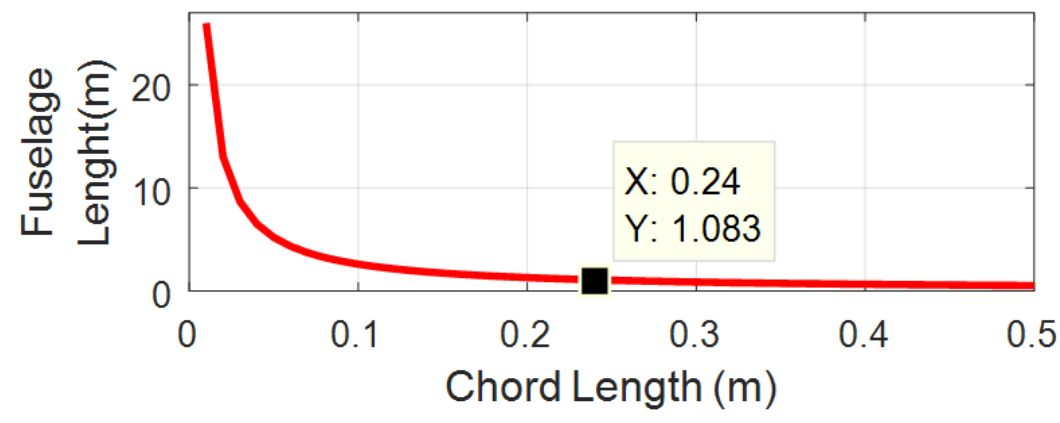

Fig. 6. Chord length against fuselage length for $3.0 \mathrm{~kg}$ lift-off mass UAV

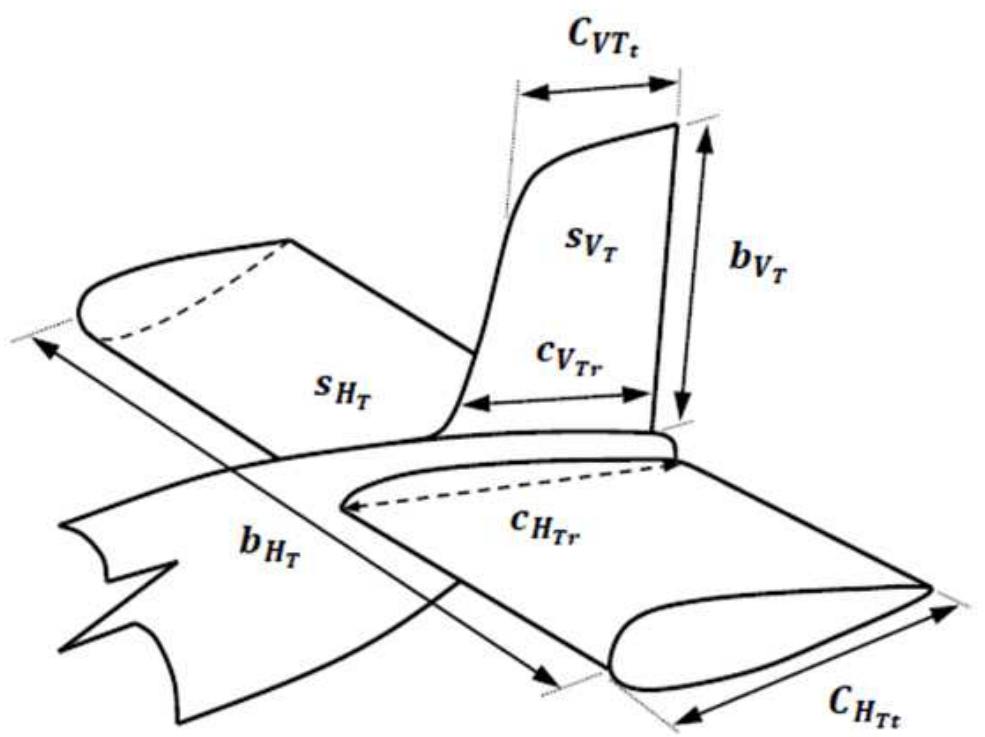

Fig. 7. Aircraft empennage 


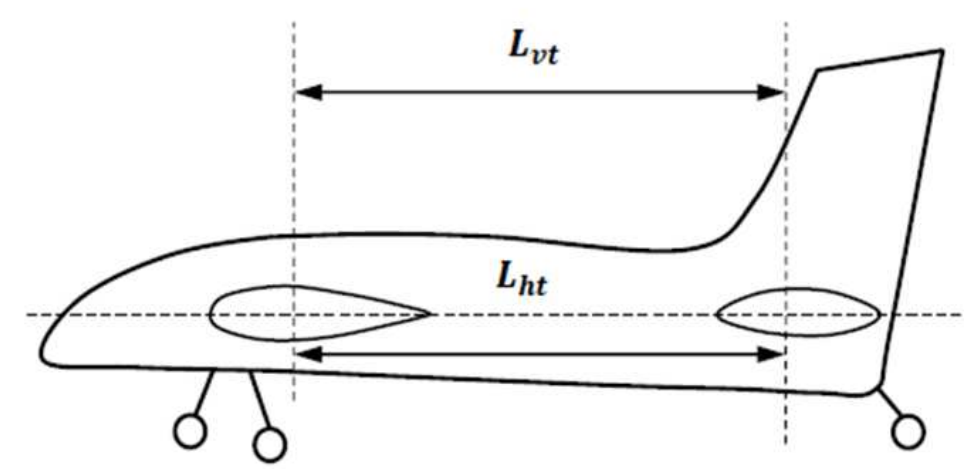

Fig. 8. Horizontal tail arm $\left(L_{H T}\right)$ and Vertical tail arm $\left(L_{v T}\right)$ of an aircraft
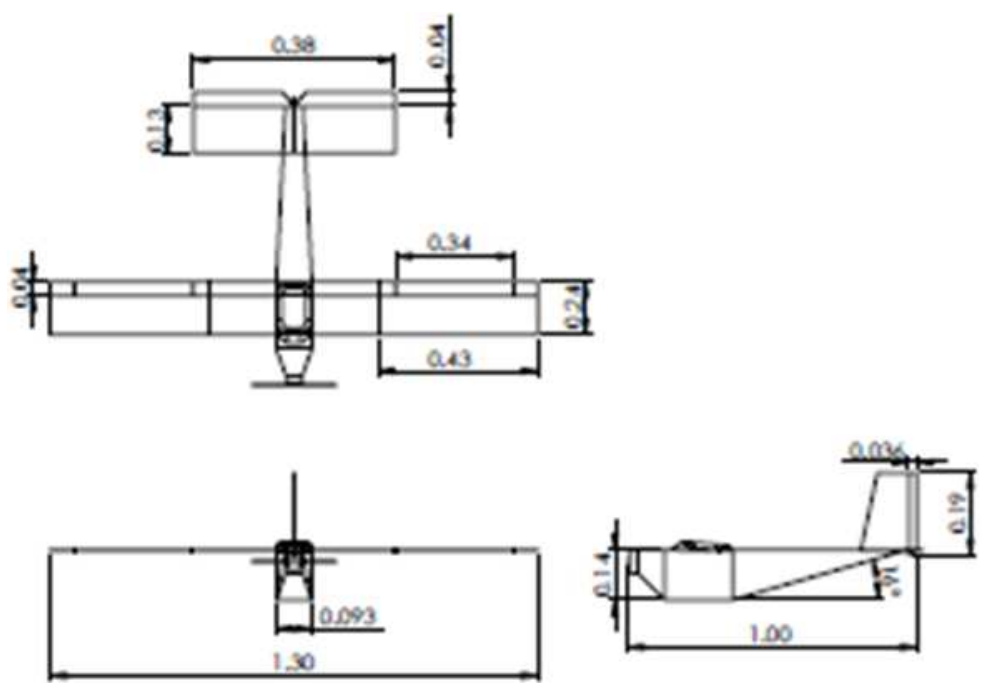

Fig. 9. Structural layout of the UAV

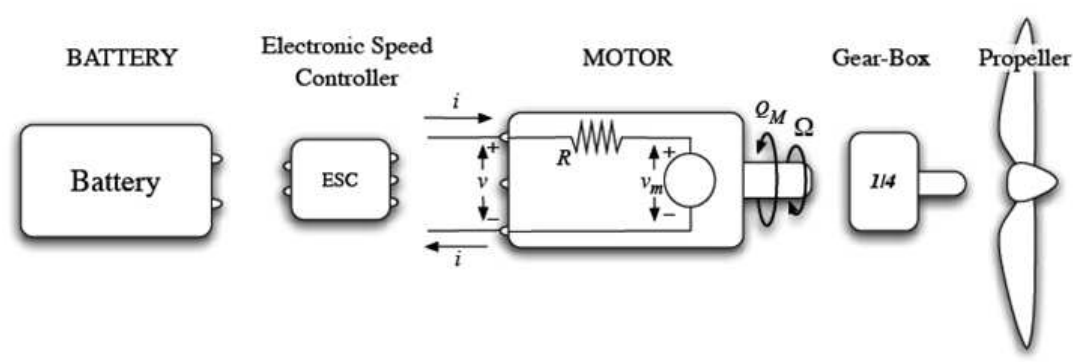

Fig. 10. Propulsion System of the UAV

Without any telemetry and payload instrumentation system on the UAV, the weight of CSTP_UAV_3.0_I for conceptual 1 flight tests was $1.6 \mathrm{~kg}$. From Fig. 13, it can be seen that the UAV attained lift-up at a short take-off distance as expected. Also, excessive fluttering of the wings was noticed after flight tests. This observation prompted an investigation that lead to the novel expressions in
(18) and (20). To obtain a shorter wingspan but larger chord length for a designed take-off mass of $3 \mathrm{~kg}$, we came up with various prototypes that could be tested for minimal fluttering of the wings. In Table 6, we propose just three of such variants. These variants of the UAV prototypes were obtained from Fig. 5 (chord length against wingspan) and Fig. 6 (chord length against fuselage length). 


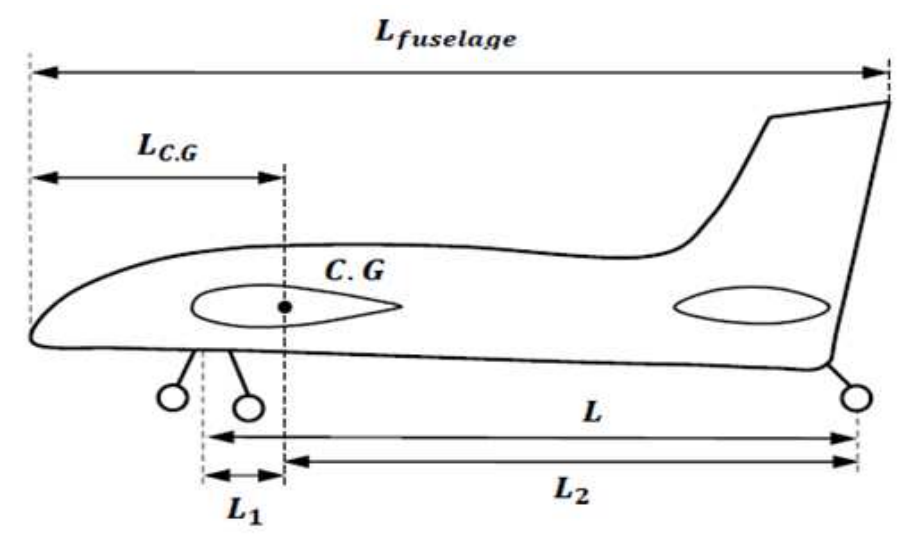

Fig. 11. Position for landing gear

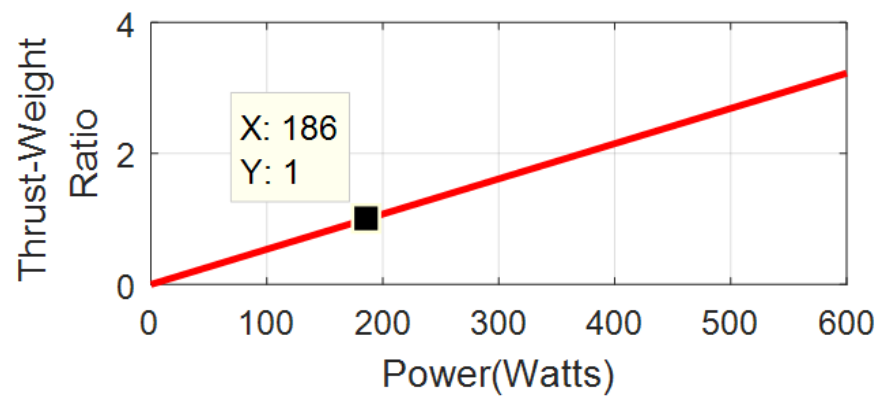

Fig. 12. Power against thrust-weight ration

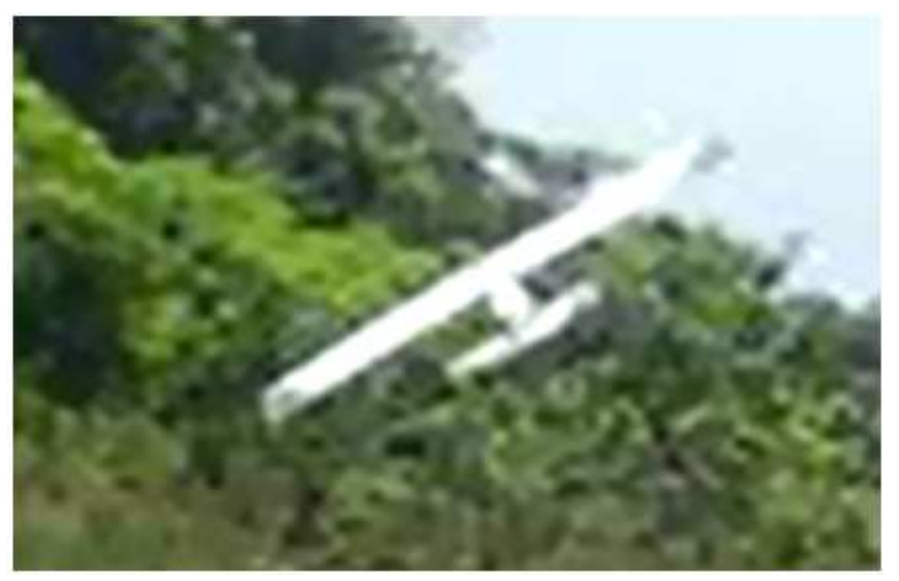

Fig. 13. CSTP_UAV_3.0_I

Table 6 . Variants of $3 \mathrm{~kg}$ lift-off mass UAV prototype

\begin{tabular}{lllll}
\hline S/N & UAV & Wingspan $(\mathrm{m})$ & Chord length $(\mathrm{m})$ & Fuselage length $(\mathrm{m})$ \\
\hline 1 & CSTP_UAV_3.0_II & 1.1 & 0.30 & 0.9 \\
2 & CSTP_UAV_3.0_III & 0.9 & 0.35 & 0.7 \\
3 & CSTP_UAV_3.0_IV & 0.8 & 0.40 & 0.6 \\
\hline
\end{tabular}

\section{Conclusion}

Mission statement for designing an aircraft for the sole purpose of surveillance in Oil and Gas pipeline network was translated to a physical UAV design. This was aided by; firstly, (3) novel mathematical expressions that relates the lift-off mass of an aircraft with the wingspan, chord length and fuselage length of the 
aircraft. Secondly, for a designed take-off mass, two (2) novel equations were used to relate the chord length with the wingspan and fuselage length of the same aircraft. These last two novel equations make it possible to fabricate several variant of the UAV without compromising the design lift-off mass. As such, an opportunity to optimise the design amid excessive fluttering of the wings was achieved. Also, in a new approach, computed thrust-to-weight ratio of the UAV during cruise flight with values greater than one unveiled the minimum power requirement for the entire flight mission.

\section{Acknowledgement}

The authors will like to acknowledge the invaluable support from the entire staff of CSTP especially those in the Aircraft/UAV unit.

\section{Funding Information}

This work was fully supported by the Federal Government of Nigeria, with grant FPN30764870032222133.

\section{Author's Contributions}

Aliyu Bhar Kisabo: Designed the study, formulated the five-novel mathematical aircraft equations and drafted the manuscript.

Charles Attah Osheku: Formulated the novel thrustto-weight ratio equation, edited the drafted manuscript, supervised the entire study and secured its funding.

Sholiyi Olusegun Samuel: Carried out the ESC programing experiment, assisted in result interpretation and managed literature searches.

\section{Ethics}

Authors declare that there are no ethical issues regarding this publication.

\section{References}

Ashok, G. and M.S. Selig, 2001. Low-speed naturallaminar-flow airfoils: Case study in inverse airfoil design. J. Aircraft, 38: 57-63. DOI: 10.2514/2.2734

Austin, R., 2010. Unmanned Aircraft Systems: UAVs Design, Development and Deployment. 1st Edn., Wiley, Chichester, ISBN-10: 0470664800, pp: 372.

Brezina, A.J. and S.K. Thomas, 2013. Measurement of static and dynamic performance characteristics of electric propulsion system. Proceedings of the $51 \mathrm{st}$ AIAA Aerospace Sciences Meeting including the New Horizons Forum and Aerospace Exposition, Aerospace Sciences Meetings, (ASM' 13).

Bronz, M., 2014. A contribution to the design of long endurance mini unmanned aerial vehicles. $\mathrm{PhD}$ Thesis, Universite de Toulouse.
Brusov, V. and V. Petruchik, 2011. Design approach for selection of wing airfoil with regard to micro-UAVs. Proceedings of the International Micro Air Vehicles Conference, (AVC' 11).

CPSU, 2013-2014. California Polytechnic State University, San Luis Obispo. 2013-2014 AIAA UAV Design/Build Fly Report.

Drela, M., 2005. Dc motor and propeller matching, lab 5 lecture notes. Technical Report, MIT.

Drela, M., 2007. First-order DC electric motor model. Technical report, MIT, Aeroand Astro.

Giuseppe, L., 2008. Aerodynamic and structural design of a small Nonpolar wing UAV. MSc Thesis. University of Dayton.

Gomez, C. and R.G. David, 2010. Small-scale airborne platforms for oil and gas pipeline monitoring and mapping.

Gur, O. and A. Rosen, 2009. Optimizing electric propulsion systems for unmanned aerial vehicles. J. Aircraft, 46: 1340-1353. DOI: 10.2514/1.41027

http://www.motocalc.com/motodown.htm

http://www.profili2.com/eng/prices.asp

Idachaba, F.E., 2013. SPDC- Pipeline Association of Nigeria Conference Port Harcourt-Nigeria.

Jenkinson, L.R. and J.F. Marchman, 2003. Aircraft Design Projects: For Engineering Students. 1st Edn., Elsevier, Burlington, ISBN-10: 0080498957, pp: 400.

Onwuka, E. and O.N. Dike, 2015. Effect of surveillance on oil pipeline vandalism in the downstream sector, Nigeria. European J. Bus. Soc. Sci., 4: 1-9.

Rutkay, B.D., 2014. A process for the design and manufacture of propellers for small unmanned aerial vehicles. PhD MSc Thesis, Carleton University Ottawa, Ontario.

Sadraey, M., 2010. Chapter 5 wing design, Daniel Webster College, MSc Thesis.

Scorpion SII-2215-1810 Motor Propeller Data: i2,229 data chart

Selig, M.S., 1995. Summary of Low-Speed Airfoil Data. 1st Edn., SoarTech Publications, Virginia, ISBN-10: 0964674718, pp: 317.

SIG Mfg. Co., Inc Catalog 66.www.sigmfg.com

Struett, R.C., 2012. Empennage sizing and aircraft stability using mATLAB ${ }^{\circledR}$. Senior Project Presented to The Faculty of the Aerospace Engineering Department California Polytechnic State University, San Luis Obispo.

TGC, 2011. Teal group predicts worldwide UAV market will total over $\$ 80$ billion in its just released 2010 UAV market profile and forecast. Teal Group Corporation.

Youngren, H. and M. Chang, 2011. Test, analysis and design of propeller propulsion systems for MAVs. Proceedings of the 49th AIAA Aerospace Sciences Meeting Including the New Horizons Forum and Aerospace Exposition, Aerospace Sciences Meetings, (ASM' 11). 